\title{
A Programming Workshop using the Robot "Thymio II": The Effect on the Understanding by Children
}

\author{
Stéphane Magnenat, Fanny Riedo, Michael Bonani, and Francesco Mondada
}

\begin{abstract}
Robots are both fascinating objects for the general public and devices whose conception, understanding and programming involve many fields. This unique combination makes them an ideal tool for introducing science and technology to children. This paper presents the outcome of a programming workshop held on the occasion of the 2011 EPFL Robotics Festival. This workshop introduced programming using the robot "Thymio II". The participants enjoyed this workshop very much, and their attitudes suggest that the public is attracted to such events out of interest rather than pure fun or educational concerns. Children appreciated the supervision, characterized by a high staff-per-child ratio of $1 / 3$. We also show that in an hour of tutorial, children were able to acquire concepts such as the sensor or the loading of a program on the robot because they practised these enough. More theoretical and less practised concepts, such as the sensory-motor loop or the programming details, were not well understood. These findings now enable us to create better edutainment material.
\end{abstract}

\section{INTRODUCTION}

Robots are at the same time fascinating objects for the general public and devices whose conception, understanding and programming involves many fields. This unique combination makes them an ideal tool for introducing science and technology to young generations. Yet what children do comprehend through their robot manipulation has not been well established. This paper aims at evaluating the impact of such manipulations in order to increase the efficiency of robots as educational tools in the future.

Scientific and technical education using robots can take place in formal or informal environments. Formal educational environments are mainly provided by schools, while informal and semi-formal educational environments can be found at home, in festivals, camps, etc. Since 2008, our lab (LSRO) has been organizing an annual Robotics Festival at the École Polytechnique Fédérale de Lausanne (EPFL). Within this festival, we propose various activities such as presentations, demonstrations and workshops. The last edition of the festival took place on May 7th, 2011. This one-day event was attended by 13000 persons and hosted 28 workshops. Workshops provide a semi-formal educational environment that allows children to improve their understanding of the different robotic

This research was supported by the Swiss National Science Foundation through the National Centre of Competence in Research Robotics. We thank the reviewers for their insightful comments, Emmanuel Eckard for the pictures of the festival and Ève Lasserre for proofreading the manuscript.

S. Magnenat is with the Autonomous Systems Lab, ETH Zürich, Switzerland: firstnamedlastname.net. F. Mondada and F. Riedo are with the Laboratory of Robotic Systems, École Polytechnique Fédérale de Lausanne, Switzerland firstname.lastnamedepfl.ch. M. Bonani is with Mobsya, Lausanne, Switzerland: firstname. lastname@mobsya.org technologies: mechanics, electronics, computer science, system integration, etc. One of these workshops was devoted to programming a mobile robot. At the end of this workshop, we asked the participants to fill a survey, covering two main themes: the appreciation of the workshop and the technical understanding of robots' functioning and programming. We report in this paper the results of the survey and discuss the lessons learnt for similar workshops in the future.

\section{RELATED WORK}

There already exist many robots intended for children to discover robotics. Among the commercially available platforms, the most well-known is probably the LEGO Mindstorms [1]. Children can build this modular robot and program it with a graphical language. There is also a large amount of didactic material available. Another successful platform, despite its extreme simplicity, is the TTS' Bee-Bot [2]. It is programmable with buttons only: the robot can register a sequence of directional orders and execute it. A more complex platform, Parallax' Boe-Bot ${ }^{1}$ is interesting to learn how to build a robot, with some notions of programming and electronics. It is especially adapted to beginner hobbyists [3], [4]. Parallax' Scribbler on the contrary is a complete robot that can be used to make drawings and basic programming [5]. Our workshops are devised to facilitate the understanding of the robot's functioning, with an emphasis on the sensory-motor loop. The existing platforms do not meet our requirements, for they have too few sensors and do not offer enough direct possibilities to visualize the internal state of the robot.

There is a need to measure the impact of robots on children's learning. We know that robots trigger enthusiasm and can be introduced successfully in school programs. For example, a wide program set up in Piedmont allowed to bring different robotic platforms into schools in a coherent way. Teachers in collaboration with university researchers created a school network to provide a framework and materials for teaching with those robots [6]. They now have more than 100 teachers involved and work on 4 different platforms (Bee-Bot, Scribbler, Mindstorms RCX and NXT) with a programming language adapted to children. Several other initiatives involving robots were launched to arouse children's interest in technical fields. The fascinating aspect of robots, along with an attractive interactive behaviour, could help to involve children who would not otherwise be very motivated by technical questions. A good example of this type of action is the Roberta Initiative [7], which aims at making robotics

\footnotetext{
1 a different robot from a another manufacturer than TTS' Bee-Bot
} 
accessible to all children, with a special emphasis on not discriminating girls. Roberta centres train robotics teachers and organize gender-sensitive robotics courses based on the LEGO Mindstorms. Based on a similar experience, Rusk and Resnick [8] give advice to broaden the participation to robotics workshops, courses and exhibitions.

Unfortunately, many studies lack quantitative analyses and concentrate on the enthusiasm robots bring without measuring the real impact of most initiatives. Some studies describe in a qualitative way the influence the robot has on the children's learning. Among the few that do describe in a qualitative way the influence of the robot manipulation on children's learning process, Highfield's study focuses on how robots can help introducing some mathematical concepts at an early age [9]. However this experience was carried out with few children over a short period of time, and the conclusions are yet to be confirmed by the next phase of the study, which will consist in longitudinal case studies with more children. In Japan, researchers tried to determine if attending some robotics lessons helped children to understand general technical problems [10]. While promising, this type of studies is still rare. In a US military academy, a real quantitative analysis was performed over the year 2000-2001 [11]. The results show that the manipulation of robots created an opposite effect to the expected one: students having taken the course with robots were compared to others who had been taught the same subjects but without robots. The students who used robots performed unequivocally worse than the others on the tests, and it did not influence their choice of discipline towards technical fields. This failure might be due to the restricted access to the robots outside lab hours, that prevented the students from debugging and experimenting. Nevertheless, this shows that we cannot measure the success of robots as educational tools by stopping at the obvious enthusiasm encountered. We need to determine what robots can bring, which concepts are better illustrated and understood, but also what are the key parameters in the context of the courses and the platform itself. These range from coaching to the possibility for children to practise outside the courses.

\section{Materials AND MethodS}

\section{A. Workshop}

The workshop we report in this paper was devoted to programming the Thymio II robot. We held 4 sessions of 1 hour 20 minutes each, with about 30 participants per session. Participants had to register before the workshop and pay $59 \mathrm{CHF}$ in advance for the robot, which they could take home afterwards. The first 5 minutes were dedicated to ticket checking and installation, and the next 15 minutes to a short presentation. This presentation consisted in an overview of the robot (LEDs, distance sensors, motors) and of the Aseba development environment. In the remaining hour, the participants programmed by themselves, following a tutorial ${ }^{2}$ and supervised by assistants, at a ratio of one assistant per three attendees. Fig. 1 shows some pictures shot

\footnotetext{
${ }^{2}$ https://aseba.wikidot.com/en:thymiotutoriel
}
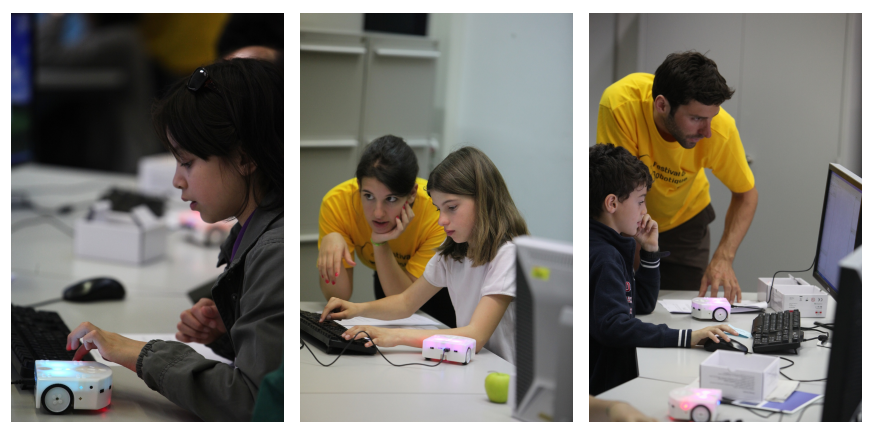

Fig. 1. Some photos of the workshop

during the workshop. At the end of the workshop, we asked the participants to fill a survey, from which we extracted the data presented in this paper.

\section{B. Survey and Statistics}

Table I shows the English translation of the survey. This survey has three parts. The first part asks general information about the participant's profile. The second aims at measuring the appreciation: of the workshop in general, but also of the hardware, and the quality of supervision. The participants are also asked whether they would apply adjectives such as "educational", "funny" or "interesting" to the workshop. Additionally, we ask them how they came to the workshop (how they found out, previous attendance). The last part aims at identifying what the participants were able to learn during this workshop. It holds 7 questions: the first targets the change in the perception of robotics thanks to the festival as a whole, while the next 6 questions focus more specifically on robotics and programming knowledge. In the translated version of the survey presented in this paper, we have marked the correct answers with a tick $\checkmark$.

To quantitatively compare the different variables of the survey, we use the Spearman's rank correlation test, and all the p-values refer to this test. We chose this non-parametric test of the monotonic dependence for our survey's entries lie in different spaces, such as age and gender. A monotic relation is the easiest to see and explain; when evoking a non-monotonic relation, we will refer directly to the plot.

\section{Thymio II Robot}

Thymio II is a miniature $(10 \mathrm{~cm})$ differential-wheeled robot designed for education. It is the result of several iterations of development and tests with children, as described in [12]. It is best used on a table top, beside a computer. In order to provide rich interaction possibilities, its main body is made of translucent white plastic and features many LEDs that indicate the status of the robot's sensors. These LEDs can also be controlled by the programmer. Fig. 2 shows a diagram with a list of Thymio's actuators and sensors while Fig. 1 shows the robot being used by children. Thymio II runs the Aseba software framework (see next section). The part of the survey called appreciation shall evaluate the robot and the workshop's approach. 
TABLE I

THE SURVEY FORM

\section{Profile}

- birth date

- gender

- Would you have preferred a boy-only/girl-only workshop? (yes/no)

Appreciation

- Give a general appreciation grade to this workshop (1 to 6)

- Give a grade to the hardware (1 to 6)

- Give a grade to the supervision (1 to 6 )

- How would you characterize this workshop? (educational, funny, interesting)

- How did you choose this workshop? (multiple answers possible) (discussion with friends/family, description, discussion with festival staff, price, duration, availability)

- Have you already taken part in other workshops? (no, this year, in 2010 , in 2009 , in 2008)

\section{Learnt concepts}

- Were you surprised by the robots you saw today?

- Yes, they are able to do things I thought impossible for a robot

- Yes, they are unable to do things I thought possible for a robot

- Yes, because they are different from other robots

- No, they are like I imagined them

- No, I already saw them

- What is a robot exactly?

- An automaton that runs a program moving it regardless of the outside world

- A device that measures physical values and allows to visualize them

- A device able to perceive the world, and to react given what it has seen $\checkmark$

- A mobile device that randomly moves in function of pre-defined parameters

- What are the characteristics of a sensor?

- It measures things around the robot $\checkmark$

- It shows what the robot does

- It allows the robot to change its environment

- It tells the robot what is around $\checkmark$

- It allows robots to communicate with each other

- At which distance can Thymio II detect obstacles?

- I do not know

- When it touches them

- At $2 \mathrm{~cm} \checkmark$

- At $5-10 \mathrm{~cm} \checkmark$

- At $10-50 \mathrm{~cm}$

- At more than $50 \mathrm{~cm}$

- How does Aseba allow to program a robot?

- The robot sends information to the computer, that handles it, and that sends commands to the robot

- The robot controls the computer; depending on what the robot perceives, the computer runs different programs

- I was not able to program the robot using Aseba, therefore I do not understand how it works

- I write the program on the computer, and the program runs on the robot $\checkmark$

- What is a variable?

- An object from the world, like a table, a chair, a wall

- A value in the robot's computer that can represent something from the world or something abstract $\checkmark$

- A number, like 3 or 9.81 , that represents a physical constant, like gravity

- A text in Aseba Studio, that is replaced by constants when the program is sent to the robot

- What is a if?

- A word that tells the robot to take a decision in function of the value of a variable $\checkmark$

- An English word that I do not understand, I would like Aseba to speak French

- A word that allows to put a value inside a variable

- A word that allows to execute many times the same program part

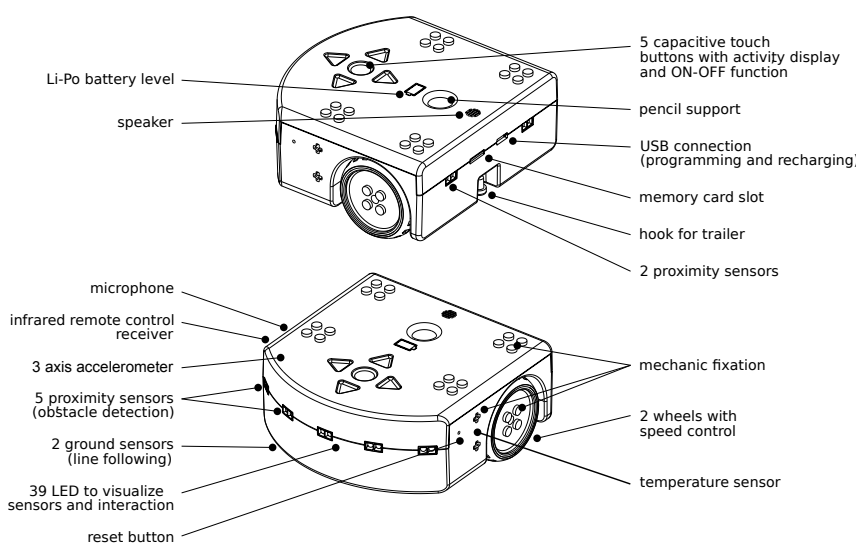

Fig. 2. Thymio II's sensors and actuators

\section{Aseba Environment}

Aseba [13] is an open-source software framework designed to enable novices to program robots easily. On the robot side, Aseba provides a lightweight virtual machine, tiny enough to run on microcontrollers like the PIC inside the Thymio. Using a virtual machine allows instantaneous upload and safe execution of code. On the desktop side, Aseba provides an integrated development environment (IDE) which allows to program in a user-friendly scripting language (Fig. 3). This IDE provides on-typing compilation and editing aids like colour syntaxing.

The Aseba scripting language is a simple, imperative, matlab-like language providing integers and arrays of integers as data types. Common logical and mathematical operations are available as operators, and additional mathematical functions such as trigonometric ones are available as native functions. These are functions implemented in native code and accessible from the virtual machine. In Aseba, code blocks are associated to events, simplifying the writing of real-time behaviours. Events are triggered by the robot's sensors or actuators. The value of the sensors and actuators are available through pre-defined variables and native functions, which are robot-dependent and therefore enumerated dynamically by the robot when the IDE connects.

We expect the facilities that Aseba provides to allow text-based programming with reasonably young children, starting from around 11 years old, depending on the available coaching. The last part of the survey, called learnt concepts, shall test what the children actually understood through their workshop experience. On previous festival editions, we ran a similar workshop, only with simulated robots [14].

\section{Results AND Discussion}

\section{A. Profile of Participants}

A total of 116 participants took part in the workshop and filled the survey. Most are children between 8 and 14 years old (Fig. 4, top), only one participant is above 20 (44), 2 did not give their age. 91 participants are boys, 22 girls, and 3 unknown. The distribution of gender slightly varies with age, with a trend of having more girls at younger ages 


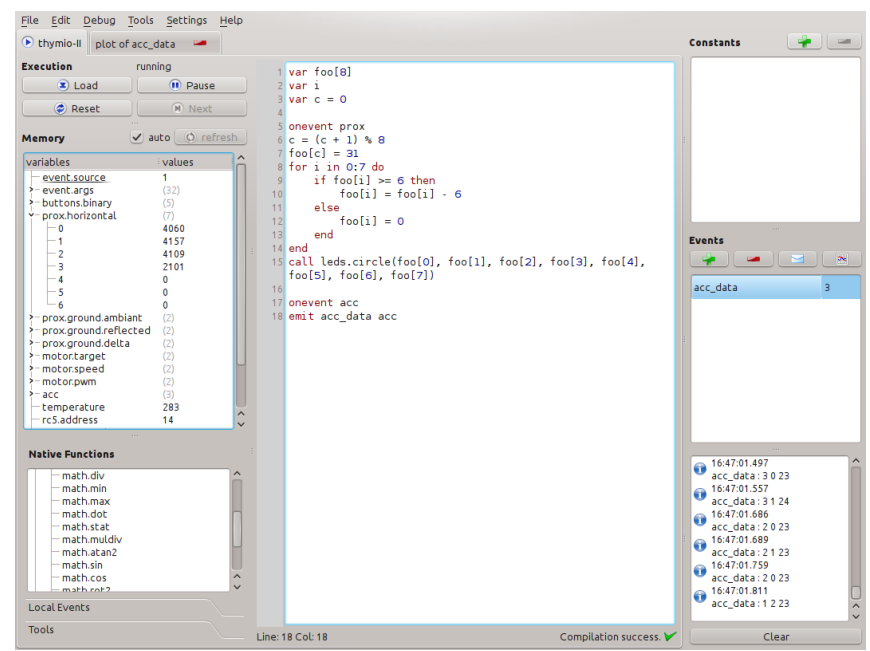

Fig. 3. Aseba Studio, the integrated development environment
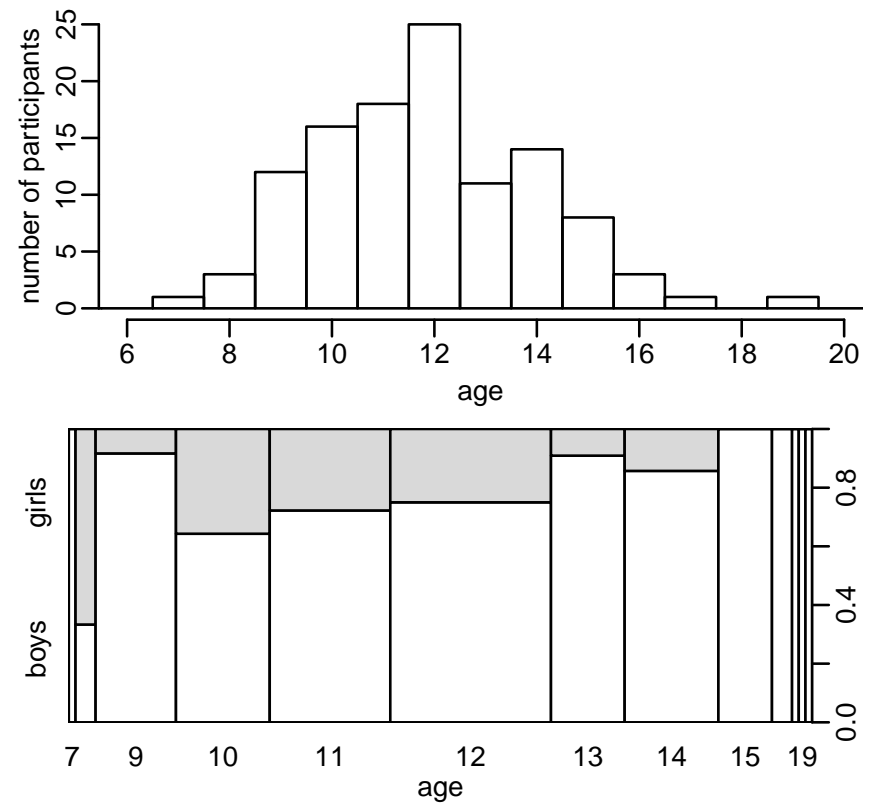

Fig. 4. Distribution of ages (top) and gender in function of age (bottom)

(Fig. 4, bottom, p-value=0.04). To understand this, Fig. 5 shows the general appreciation in function of age for girls. This appreciation does not correlate with age ( $\mathrm{p}$-value $=0.48$ ). Therefore, we attribute the decreasing number of girls with age to the prejudice in our western societies against girls in technical fields, which would affect the decision of older girls and their parents, although we might assume that the interest in robotics should a priori be even for boys and girls.

\section{B. Appreciation}

The general appreciation is very high: the worst note is 4 (10 participants) on a scale of 1 to 6 , while 67 participants (57\%) give 6. Two participants failed to answer this question. Fig. 6 shows the appreciation in function of age and gender. Table II shows the p-values of the correlation test between the different appreciations and the age, gender, and adjectives.

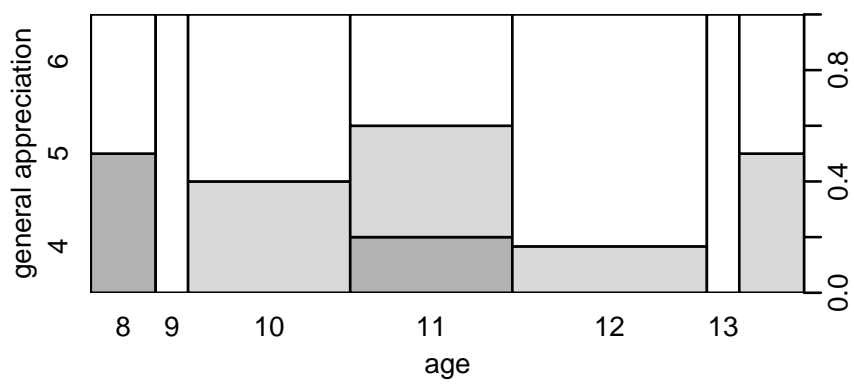

Fig. 5. Appreciation in function of age for girls
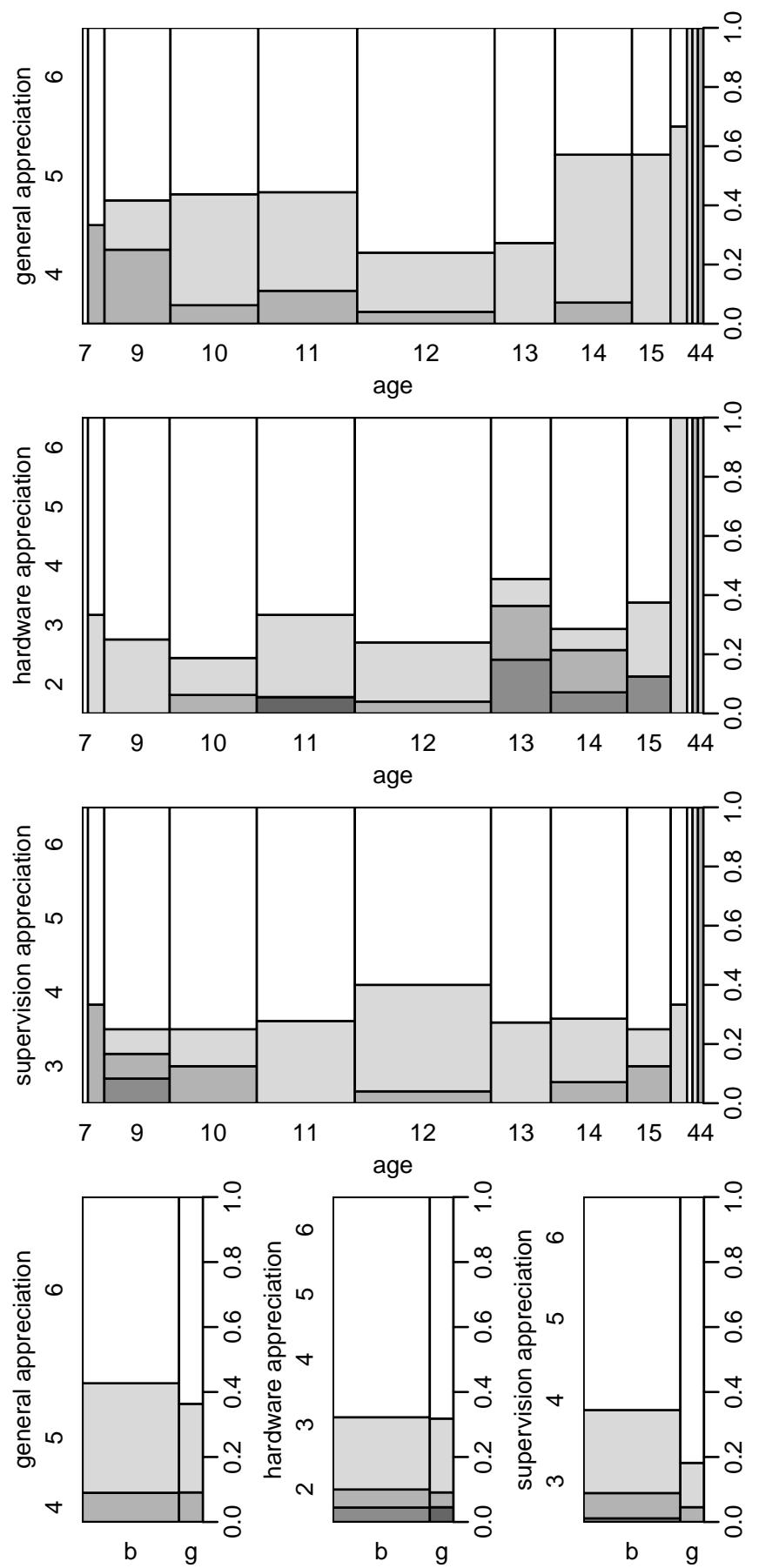

Fig. 6. Appreciation in function of age and gender 
TABLE II

P-VALUES OF SPEARMAN'S RANK CORRELATION TEST

\begin{tabular}{l|ll|lll}
\hline appreciation & age & gender & interesting & funny & educational \\
\hline general & 0.44 & 0.63 & 0.004 & 0.997 & 0.88 \\
hardware & 0.03 & 0.97 & 0.59 & 0.54 & 0.33 \\
supervision & 0.60 & 0.15 & 0.23 & 0.67 & 0.37 \\
\hline
\end{tabular}

The general appreciation correlates neither with age nor with gender. It strongly correlates with interesting but not with funny or educational. This shows that children came to the workshop primarily by interest, and not only to have fun or because they actively want to learn something. For us, this means that Thymio II has a high edutainment value, being at the right place between fun and seriousness. The appreciation of hardware is also high, since 78 participants $(67 \%)$ give 6 , while only 12 participants $(10 \%)$ rate it under 5 , and one participant did not answer this question. Hardware appreciation is gender-neutral but correlates with age: younger children like the hardware more than the older. Young children are thrilled by the many LEDs distributed over Thymio's body. Older children might be a bit frustrated by the relatively simple body. At last, the appreciation of supervision is the highest, with 80 of the participants $(69 \%)$ who give a 6 and only $9(8 \%)$ of them who rate it below 5; one did not answer this question. Supervision appreciation is age-neutral and is slightly more appreciated by girls than boys, but not by a statistically significant amount. This shows that the high staffper-child ratio of $1 / 3$ is a good choice. We tried to balance the gender of the staff, which might also have positively affected the rating from girls. Neither the hardware nor the supervision appreciations correlate with any adjective.

\section{Learnt Concepts}

The last 6 questions of the survey provide an insight into the participants' understanding of the different technical concepts. For each question, the answer can be correct, wrong, or invalid (for instance if the question was not treated or if multiple answers were given). We compute a grade by adding one point for each question with a correct answer. The grade histogram (Fig. 7, top) shows a bimodal distribution with a median at 3 . The peak at 0 is explained by the fact that 17 participants did not answer these last 6 questions. The grade correlates with age $(p$-value $=0.02)$ and with gender $(p$-value $=0.04)$ : older children and boys have better grades than younger children and girls. We attribute the better score of boys vs girls to a greater familiarity of boys than girls with technical-related activities, which we think is presumably due to a bias in society and therefore in parents' decisions with respect to gifts, books and games.

The general results show that the participants have not really understood what a robot is, since 33 participants $(28 \%)$ only gave the correct answer and 37 (32\%) of them thought that it was an automaton that moved regardless of the outside world (Fig. 7, table). This might be partially due to the confusion created by the other so-called robots present at the festival, many of them only automatons, but also to the complexity of the question and its use of the phrase "runs a program". This probably lead many children to think that, since the robot does run a program, this proposition must be the right one. On the whole, the concept of sensory-motor loop has not been understood. It is therefore important in the future to render this concept more concrete and possible to be practically experienced, because the sensory-motor loop is the most important concept in autonomous robotics.

On the contrary, the concept of sensor is well understood, since 78 participants $(67 \%)$ correctly answered the general question about sensors and $66(57 \%)$ chose the right answer about the distance at which Thymio II can detect obstacles. The reason for this success might be the close interaction between the child and the robot that playing with this sensor requires. By imagining that she or he is the robot, a child can build her or his own image of how the robot sees the world, and therefore understand the concept of sensor. Moreover, in the tutorial, the children are told to test the sensors and see how far they can detect things. The concrete use of a sensor through practical exercises most probably help the children to understand the concept of sensor.

The $73(63 \%)$ correct answers to the question about programming indicate that the participants also understand well how Aseba allows to program robots. This is probably due to the focus we put on the description of the Aseba architecture in our introductory talk, so that the children do not feel lost in the complexity of the system. It is heartening to see that children can understand some of the architectural notions of a relatively complex system, if it is sufficiently well described and experienced. Indeed, practise may again play a critical role, since the children have to click on the "load" and "run" buttons each time they want to run their code on the robot, which is a very concrete approach of Aseba.

The technical questions related to programming, about the notion of variable and the if instruction, respectively obtain $39(34 \%)$ and $48(41 \%)$ correct answers, which suggests that this aspect is not well understood. The tutorial introduces the if instruction but does not explicitly practise it. We should improve this, for instance by using the integrated debugger and executing a program step-by-step with and without an obstacle in front of the robot, in order to visualize the execution flow. The notion of variable is only implicitly defined, and therefore not acquired by the children. Furthermore, the short time available for programming ( 1 hour) certainly affects these results. Indeed, the children were really motivated to advance in the tutorial, and sometimes copied/pasted lines of code without taking the time to understand them. However, as children do take the robot home with them, we expect the most interested to further explore programming concepts.

\section{CONCLUSION}

Our programming workshop with Thymio II was globally very much appreciated, regardless of age and gender. The hardware appeals to children, both to girls and boys. Yet what the children appreciated the most was the supervision, which with one assistant per three children was well above school standards. This shows once again the importance of having 

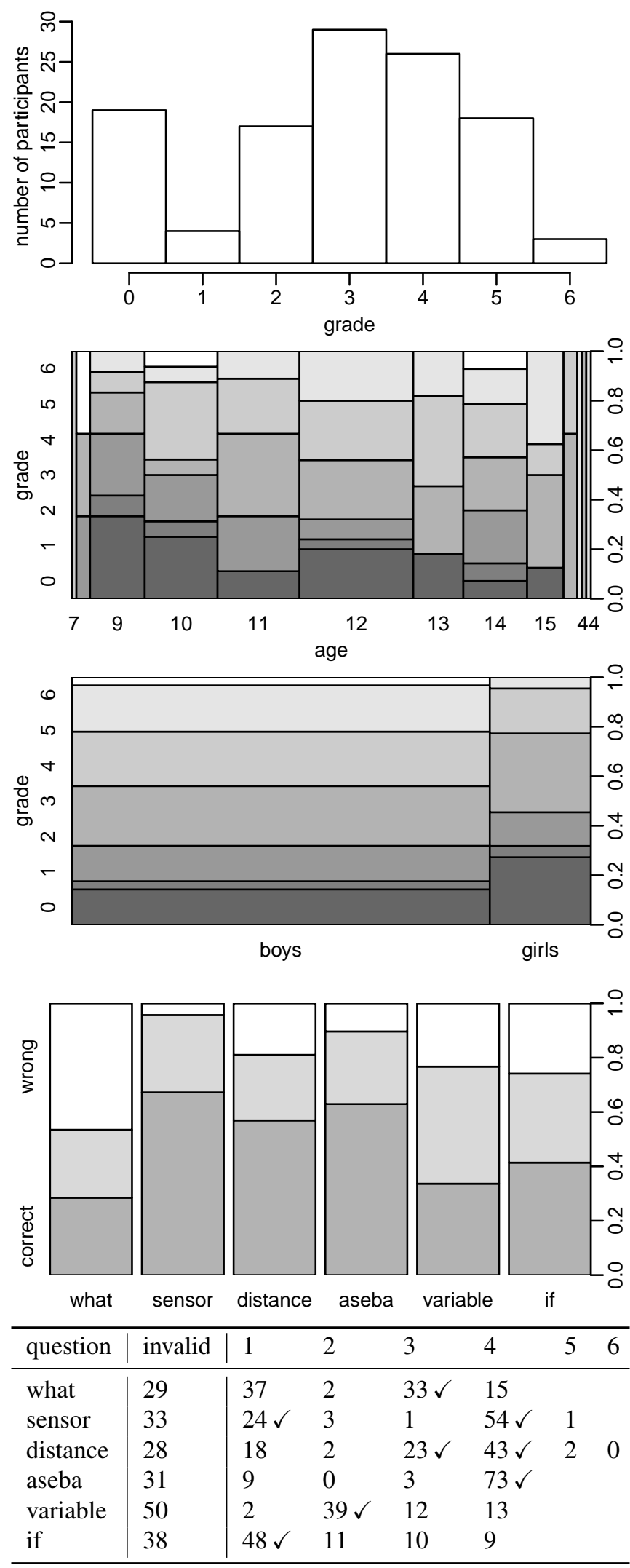

Fig. 7. Understanding in function of question, age, and gender. In the bottom plot, the area between correct and wrong corresponds to invalid entries. The table gives the frequency of each possible answer for each question. enough staff for quality education. Children come to such a workshop primarily by interest, and not only to have fun or because they actively want to learn something. Nevertheless, they enjoy the workshop and do learn new things, at the level of practical elements in particular, such as what a sensor is and what it can perceive. Children are also able to understand a relatively complex system like the Aseba framework, as long as they practise it long enough. The more theoretical and less practised aspects, such as the sensory-motor loop or the programming details, are not clearly apprehended. In similar workshops, we advise the organizers to take care of introducing these concepts with quality material tailored to the audience, such as cartoons for children. These findings now enable us to create better edutainment tools for introducing science and technology to young generations.

\section{REFERENCES}

[1] LEGO Corporation. (2011, November) Mindstorms web site. [Online]. Available: http://mindstorms.lego.com

[2] TTS group. (2011) Bee-Bot web site. [Online]. Available: http: //www.beebot.org.uk

[3] Parallax. (2011) Boe-Bot web site. [Online]. Available: http: //www.parallax.com/go/boebot

[4] R. Balogh, "Basic activities with the Boe-Bot mobile robot," in Proceedings of conference DidInfo, 2008.

[5] Parallax. (2011) Scribbler web site. [Online]. Available: http: //www.parallax.com/ScribblerFamily/tabid/825/Default.aspx

[6] M. Demichele, G. Demo, and S. Siega, "A piedmont schoolnet for a k-12 mini-robots programming project: Experiences in primary schools," in Workshop Proceedings of Intl. Conf. on Simulation, Modeling and Programming for Autonomous Robots (SIMPAR), 2008.

[7] A. Bredenfeld and T. Leimbach, "The roberta initiative," in Workshop Proceedings of Intl. Conf. on Simulation, Modeling and Programming for Autonomous Robots (SIMPAR), 2010, pp. 558-567.

[8] N. Rusk, M. Resnick, R. Berg, and M. Pezalla-Granlund, "New pathways into robotics: Strategies for broadening participation," Journal of Science Education and Technology, vol. 17, no. 1, pp. 59-69, 2008.

[9] K. Highfield, J. Mulligan, and J. Hedberg, "Early mathematics learning through exploration with programmable toys," Paper submitted to PME, vol. 31,2008

[10] S. Kurebayashi, S. Kanemune, T. Kamada, and Y. Kuno, "The effect of learning programming with autonomous robots for elementary school students," in 11th European Logo Conference, 2007, pp. 1-9.

[11] B. Fagin and L. Merkle, "Quantitative analysis of the effects of robots on introductory computer science education," Journal on Educational Resources in Computing (JERIC), vol. 2, no. 4, pp. 1-17, 2002.

[12] F. Riedo, P. Rétornaz, L. Bergeron, N. Nyffeler, and F. Mondada, "A two years informal learning experience using the Thymio robot," in Proceeding of the 6th International Symposium on Autonomous Minirobots for Research and Edutainment (AMIRE), 2011.

[13] S. Magnenat, P. Rétornaz, M. Bonani, V. Longchamp, and F. Mondada, "ASEBA: A Modular Architecture for Event-Based Control of Complex Robots," IEEE/ASME Transactions on Mechatronics, vol. PP, no. 99, pp. 1-9, 2010.

[14] S. Magnenat, B. Noris, and F. Mondada, "Aseba-challenge: an opensource multiplayer introduction to mobile robots programming," in Proceedings of International conference on Fun and Games. Springer, 2008. 